\section{Cahiers de Narratologie}

Analyse et théorie narratives

$19 \mid 2010$

Images composites, arts pluriels

\title{
Les génériques et incipits des « comédies historiques » italiennes (1959-1963) : un défi de composition
}

Stefano Leoncini

\section{OpenEdition}

Journals

Édition électronique

URL : http://journals.openedition.org/narratologie/6159

DOI : 10.4000/narratologie.6159

ISSN : 1765-307X

Éditeur

LIRCES

Référence électronique

Stefano Leoncini, «Les génériques et incipits des « comédies historiques » italiennes (1959-1963) : un défi de composition », Cahiers de Narratologie [En ligne], 19 | 2010, mis en ligne le 22 décembre 2010, consulté le 20 avril 2019. URL : http://journals.openedition.org/narratologie/6159; DOI : 10.4000/ narratologie. 6159

Ce document a été généré automatiquement le 20 avril 2019.

Article L.111-1 du Code de la propriété intellectuelle. 


\title{
Les génériques et incipits des " comédies historiques » italiennes (1959-1963) : un défi de composition
}

\author{
Stefano Leoncini
}

Le cinéma est l'expression naturelle de ce siècle : c'est un art de collaboration Alessandro Blasetti, « Le cinéma : art composite ou art spécifique ${ }^{1} »$

\section{I.}

1 Le générique est, dans le film narratif de fiction, un lieu privilégié de composition iconique. Plus « composite» que l'image de cinéma ordinaire, il comporte par définition des parties scripturales, et peut faire appel à des éléments a priori hétérogènes au film en tant que support- non photographiques, notamment - même si, en dernière instance, tout sera imprimé sur la pellicule et prendra la forme unique et définitive de photogramme(s). Elément à la fois externe et interne au film, le générique entretient une indécision quant à son propre statut textuel. S'agissant du générique d'ouverture, en particulier, le déroulement (ou l'apparition, selon les cas) des «crédits» développe un discours apparemment contradictoire: si d'une part, une fois les lumières de la salle éteintes, il aménage une transition entre l'environnement réel et l'environnement spectaculaire, en s'aidant de la musique, d'autre part il maintient le spectateur dans l'attente de l'action filmique, du film communément entendu, dont il dévoile en même temps la nature intime de fiction, puisqu'il fait explicitement mention des divers corps de métier qui collaborent à sa réalisation.

2 En dépit de sa nature liminaire, le générique est donc un moment important, crucial même, du spectacle cinématographique, aussi bien au plan phatique (c'est-à-dire dans la mise en communication entre l'œuvre et son destinataire) qu'au plan esthétique. Il comporte des aspects strictement fonctionnels mais aussi, clairement, des aspects 
artistiques, ayant pu ainsi acquérir, au fil de l'histoire du septième art, une fonction plastique: aujourd'hui, des graphistes spécialisés dans le design de génériques sont célébrés comme de grands créateurs, et l'on parle volontiers de "génériques d'auteur ", quitte à séparer leur caractère artistique de la qualité du film. Sans aborder un discours trop général sur le générique de film, on rappellera néanmoins que de nombreuses recherches ont eu lieu depuis les années 90, s'intensifiant pendant les derniers temps ${ }^{2}$ et témoignant ainsi du vif intérêt porté par la postmodernité pour ce que d'aucuns ont défini comme la poétique du « fragment hybride ${ }^{3}$ ».

Avant d'entrer dans le vif de la matière, quelques réflexions d'ordre terminologique et esthétique s'imposent à propos de la notion de «composite». Dans le concept d'image composite, l'idée qui prime est celle d'un objet unique créé à partir de plusieurs objets de nature hétérogène ou provenant de supports matériels disparates. Les acceptions d'image composite que l'on peut rencontrer dans la littérature critique et théorique spécialisée comportent, la plupart du temps, l'idée d'un ajout à (d'une inscription dans) l'image ellemême d'éléments de texte et éventuellement d'éléments sonores. Mais on sort alors, visiblement, non seulement du domaine de l'iconique, mais aussi de celui du « composite »- entendu comme adoption d'un principe de supplémentarité-, pour entrer dans le domaine de la "composition"-c'est-à-dire d'une création obéissant à un principe de complémentarité. Le film de fiction est, dans sa forme la plus ordinaire, un objet audiovisuel relativement figé où la matière sonore a sa place de plein droit, et son agencement avec l'image répond à un certain nombre de règles ou de conventions, qui certes peuvent évoluer au cours de son histoire ou être transgressées dans l'acte créatif. On observera donc, dans un premier temps, dans quelle mesure la particularité du genre (ici, la comédie) influe sur la forme du générique et quelles stratégies sont adoptées pour composer une forme plus complexe que celle de départ.

\section{II.}

Les œuvres auxquelles nous nous sommes intéressés appartiennent à un corpus relativement restreint dans leur nombre et dans l'extension temporelle du filon productif qu'elles alimentent pendant environ un lustre (cf. infra: Filmographie des comédies sur toile de fond historique). Il s'agit d'un sous-genre qui a été identifié par la critique comme commedia storico-moderna ${ }^{4}$, et défini comme un "détour par l'histoire » de la comédie italienne $\mathrm{e}^{5}$. Il y est question d'une demi-douzaine de films (en réalité ce nombre double lorsqu'on y intègre des œuvres moins connues et parfois moins réussies), tous tournés entre 1959 et 1963. 1959 est l'année de sortie de La Grande Guerre de Mario Monicelli ; film primé à Venise ex aequo avec un autre film à caractère rétrospectif (Le Général della Rovere de Roberto Rossellini), il est considéré comme le prototype du genre. 1963 correspond à la sortie en salle des Camarades, aboutissement esthétique de l'expérience italienne de reconstruction de l'Histoire par le rire ou, plus exactement, par la tragicomédie. Après cette date, on continuera de tourner des comédies en costume, mais avec de tout autres intentions et sans revenir sur le contemporain, c'est-à-dire sur l'histoire de l'Italie postunitaire. Cette périodisation (1959-63) correspond assez exactement à celle qui, au plan économique et social, fut celle du "boom ", à savoir l'époque où le système industriel italien connut une expansion d'une ampleur et d'une rapidité sans précédent et sans équivalent en Europe. C'est aussi un moment politique particulier dans la jeune histoire de la République italienne où, pour la première fois, la Démocratie Chrétienne perdit des 
parts considérables d'électorat et se trouva contrainte à l'«ouverture à gauche " (expérience gouvernementale dite du centrosinistra avec l'accession au pouvoir de partis progressistes). Cet affaiblissement du parti dominant est vu comme l'occasion d'un relâchement de la censure et de l'ouverture d'espaces d'expression plus amples pour les cinéastes, qui abordent alors des thèmes jusque là écartés car considérés comme trop brûlants. Au plan de la réception et de la fortune commerciale, enfin, le cinéma italien atteint ses plus hauts sommets de reconnaissance internationale. Pour en avoir un aperçu, il suffit de passer en revue le palmarès des grands festivals cinématographiques à l'aube des années Soixante :l'année 1963 sera même, de ce point de vue, son annus mirabilis, puisque les trois plus prestigieux prix internationaux seront remportés par des films italiens. Au moins deux de ces films, Le Guépard de Luchino Visconti et Enquête sur un citoyen au-dessus de tout soupçon de Francesco Rosi, sont très représentatifs d'un génie assez typiquement italien qui consiste à savoir allier la vocation spectaculaire du cinéma à sa vocation éducative et culturelle : des éléments que le septième art porte dans son bagage génétique comme des virtualités a priori concurrentes et tendant à l'exclusion réciproque ${ }^{6}$. 


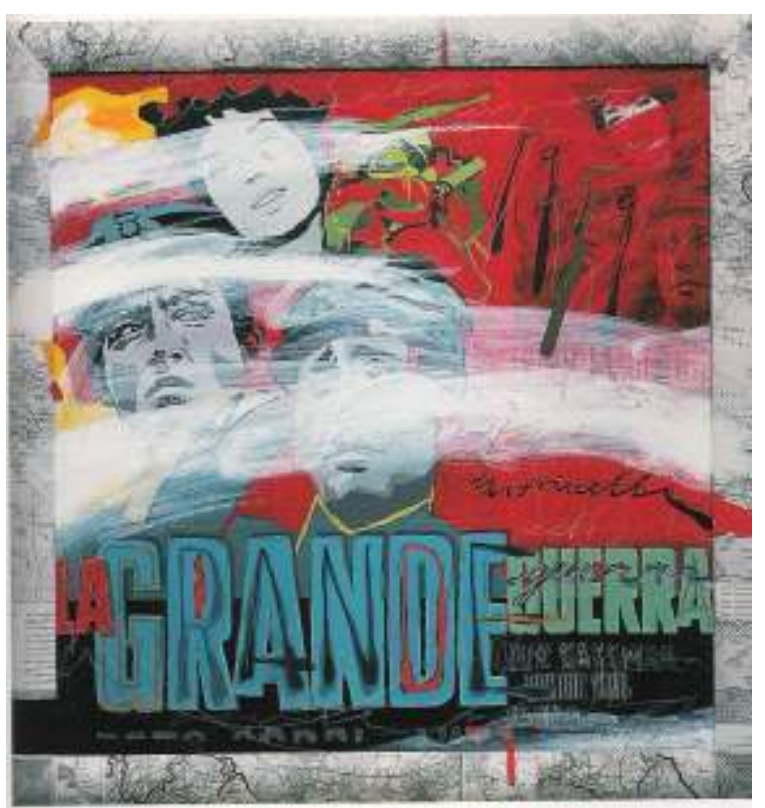

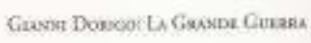

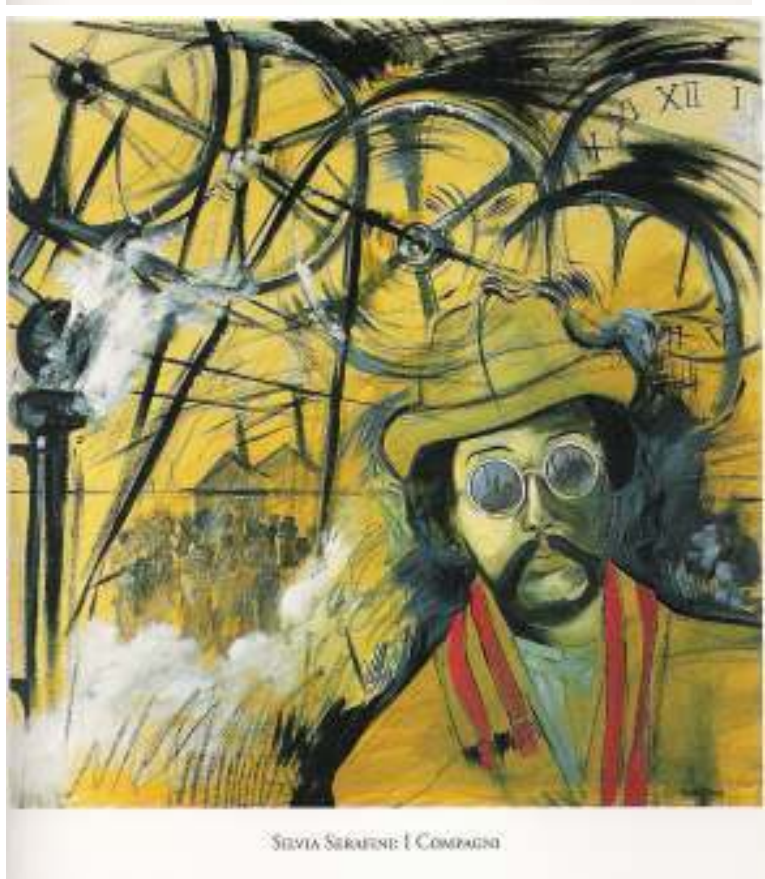

Gianni Dorigo, La grande guerra (tableau $)$; Silvia Serafini, I compagni (tableau ${ }^{8}$ )

5 La comédie historique obéit elle aussi à un principe de supplémentarité, dans la mesure où elle naît d'une exigence, fortement perçue par certains cinéastes, de contribuer au développement moral du pays, en abordant, à l'intérieur d'un genre consensuel, des thèmes que le cinéma dit « sérieux » n'avait jamais osé traiter jusque là, et cela tout en dépassant les formes génériques traditionnelles ("genres» ou "filons»). L'histoire contemporaine est le premier de ces «tabous " scénaristiques qu'il fallait tôt ou tard briser. Visconti, en décrétant le décrochage de l'actualité, est le premier parmi les «néoréalistes » à se détacher du présent et à entreprendre, dans une perspective gramscienne, l'analyse de ses raisons historiques. Senso, tourné en 1957, est le plan d'amorce d'un long travelling sur l'histoire européenne qui s'accomplira plus tard, du 
Guépard(1963) à Ludwig ou Le Crépuscule des Dieux (1972). Entretemps, c'est la comédie qui assume le rôle d'ouvre-piste, dans la conscience bien partagée parmi les cinéastes que «les Italiens [étaient] capables d'aborder tous les sujets à condition de pouvoir en rire ${ }^{9}$ ". C'est la comédie qui, la première et avec les plus de détermination et de réussite, prend en charge la mission civique de re-monumentalisation de l'histoire, appliquant, de très loin mais efficacement, la ligne culturelle de la gauche politique. Les œuvres de Monicelli, en particulier, sont probablement celles qui incarnent le mieux, tous domaines artistiques confondus, le caractère "national-populaire» préconisé à cette époque par la commission culturelle du Parti Communiste Italien : une culture socialiste par le contenu et nationale par la forme. Un véritable pari de composition à relever par les cinéastes, qui, au delà de leurs credo politiques, perçoivent très fortement cette exigence et la traduisent dans leurs œuvres, soutenus pour la première fois par la clairvoyance (ou le "flair») des producteurs, qui semblent s'être enfin persuadés que "le bon film peut chasser le mauvais ${ }^{10}$ ». Le tout, dans le contexte d'un dynamisme général de la filière, à une époque où le système productif italien est à nouveau en mesure de rivaliser commercialement avec Hollywood ${ }^{11}$.

III.

6 Si La Grande Guerre est le premier terme de cette série des comédies historiques, son générique n'a pas fait école pour les films suivants qui s'y sont pourtant référés comme à un modèle. Il est composé exclusivement de plans cinématographiques, c'est-à-dire d'images mouvantes, et plus spécifiquement de plans de détail sur des objets ou parties du corps représentant le quotidien du fantassin de tranchée. Cet agrandissement peu naturel - et en tout cas peu fréquent au cinéma, à tel point que la langue française le dénomme « insert » - introduit la dimension intimiste que Monicelli affirme avoir voulu conférer à son film. Le générique est séparé du «film» par un fondu au noir censé souligner, en l'occurrence, une absence de lien chronologique entre les deux parties de l'opus. Le dernier plan montre, comme le premier, des godillots s'enfonçant dans la boue : il ferme la boucle du générique et aménage la transition entre le régime hypo-narratif de ce dernier et le récit filmique. On remarquera toutefois que le dernier plan du générique s'enchaîne sur un élément qui n'est pas proprement filmique, ou ne l'est plus à l'époque du sonore : un « carton » (semblable à ceux sur lesquels apparaissaient les intertitres des films muets) où est inscrit le premier vers d'un chant de soldats. Le film entier est scandé par de telles césures scripturales, qui le subdivisent en "chapitres", rappelant entre autres son inspiration littéraire ${ }^{12}$. C'est ensuite par un fondu-enchaîné que le spectateur est introduit aux images de la première séquence, celle de la visite médicale, qui sera l'occasion de la rencontre entre les deux antihéros de l'histoire.

7 L'accompagnement sonore, extra-diégétique jusqu'au plan-intertitre et structuré comme un pot-pourri, joue un rôle déterminant. Les notes résonnant sur les premières images évoquent un hymne national, convoquant immédiatement la composante du patriotisme, l'un des thèmes porteurs du film. Elles s'enchaînent rapidement avec un air de mélodrame, ingrédient de base de tout le cinéma italien de l'immédiat après-guerre. Le son de trompette, symbolisant le quotidien du soldat en contrepoint aux images, ponctue d'abord la mélodie, puis s'y entremêle. La musique appuie au niveau tonal l'intimisme recherché par l'image. Jusque là, l'ensemble a des allures de rhapsodie gershwinienne, sonnant comme un hommage des auteurs de La Grande Guerre au cinéma hollywoodien. La 
fluidité de la musique permet la combinaison de plusieurs éléments additionnels, tout en conservant sa fonction traditionnelle de liant. Là où l'image introduit une césure, la musique recoud ; et lorsqu'aux accords d'orchestre, à la tonalité dramatique, se substitue par un enchaînement discret le son des flûtes entonnant un air de chansonnette, nous sommes introduits à la légèreté du quotidien, c'est-à-dire à une ambiance tonale plus propice à planter le décor de comédie.

Le générique d'ouverture de La Grande Pagaille prend un parti totalement opposé à celui de La Grande Guerre. Pour l'exposition des « crédits », les auteurs ont opté dans ce cas pour un enchaînement de photogrammes du film ou de photos de plateau, c'est-à-dire de clichés pris au moment du tournage :en tout état de cause, d'images fixes uniquement. Par ailleurs, il n'y a ici aucune hésitation à montrer par anticipation, dans ce pré-texte filmique qu'est le générique, les interprètes du film. Alberto Sordi, vedette cinématographique nationale, est montré en cadrage rapproché dès la deuxième image ; il y apparaît flanqué de Serge Reggiani, à l'époque peu connu en Italie comme comédien ; on le reconnaît à nouveau, un peu plus tard, côte à côte avec Eduardo De Filippo, autre monstre sacré de la comédie, cette fois-ci théâtrale. On sait que, pour La Grande Guerre, Monicelli aurait voulu faire appel à des interprètes plus anonymes que ne l'étaient Gassman et Tognazzi. Le contexte productif le lui permettra seulement quatre ans plus tard pour Les Camarades. L'absence de visages sur le générique de La Grande Guerre répond aussi, vraisemblablement, à cette exigence de «transparence ». La Grande Pagaille semble céder au vedettariat au moment du générique, mais son scénario et sa mise en scène sont d'une sobriété telle qu'à la vision du film l'on n'a cesse de s'interroger sur son statut

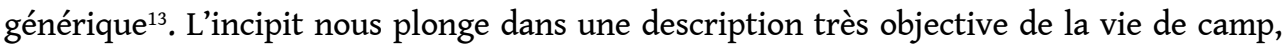
rappelant de près le style visuel des films néoréalistes. C'est paradoxalement la situation politique dans laquelle les soldats italiens vinrent soudain à se trouver, au lendemain du 8 septembre 1943, qui introduit la dimension comique, bien plus que n'y contribue la présence d'Alberto Sordi ${ }^{14}$. D'un point de vue plastique, les images fixes du générique semblent choisies en fonction du mouvement qu'elles contiennent : geste, chute, marche, course, etc., la photo étant elle-même élaborée dans ce sens. On y décèle notamment un travail sur les fumées, les vapeurs, les poussières, qui semble vouloir donner à l'image un statut indécis entre le figé et le mouvant. Une association visuelle est même suggérée entre le blanc des flous qui caractérisent les images du générique et le blanc de l'écume des vagues qui se brisent sur la plage, au premier plan de la première séquence. Au niveau musical aussi, le générique de $L a$ Grande Pagaille s'oppose assez nettement à celui de $L a$ Grande Guerre. Il s'agit d'une marche militaire assez enlevée où dominent dans un premier temps le son de la trompette et les roulements de caisse claire. L'enchaînement avec une ariette populaire couchée sur des tapis de violons, de même facture que celles que l'on pouvait entendre sur les comédies sentimentales dont Comencini, Monicelli et Risi euxmêmes étaient devenus spécialistes dans les années 50 , est presque de mise.

L'incipit de La Marche sur Rome est à considérer comme un pur chef d'œuvre de composition. Au plan de l'image, un générique composé de photos d'époque s'enchaîne avec un prélude semi-sérieux basé sur des films d'archives, chargés d'établir plus solidement le contexte. Il s'agit d'une sorte de "séquence en accolade", selon la terminologie metzienne, censée résumer la situation historique et politique dans laquelle le mouvement fasciste prend pied, commentée par une voix de narrateur. La credit sequence se greffe par un raccord plastique sur ces images d'actualité, nous plongeant dans le quotidien d'un ancien combattant revenu à la vie civile et se retrouvant au 
chômage comme la plupart de ses camarades de tranchée. Très rapidement, ce soi-disant vétéran se révèle plutôt comme un imposteur, parent proche du personnage joué par le même Gassman dans Le Pigeon, avatar ou "somme » des deux tire-au-flanc de La Grande Guerre $^{15}$. La musique qui accompagne bruyamment les images du générique est un potpourri de chants fascistes (Giovinezza, All'armi siam fascisti) et de chansons paillardes, agencés en canon, le tout assaisonné par force roulements de tambour et cris de trompette ou de cor. L'impression qui s'en dégage est celle d'une assemblée faite de bric et de broc, une image emblématique de ce que fut sociologiquement le mouvement fasciste, comme le film le raconte par une admirable série de situations offrant la scène à de nombreux personnages secondaires.

10 De même qu'il en va pour tous les autres génériques d'ouverture à partir de La Grande Pagaille, les auteurs de I compagni ont opté pour une séquence d'images fixes filmées à la caméra et montées en succession à cadence variable. Les quatre premières sont des photographies d'époque de la ville de Turin, sortes de cartes postales destinées à situer géographiquement et chronologiquement le récit (information implicite qui sera plus loin confirmée par une indication scripturale). La quatrième photo représente l'intérieur d'une usine, où des ouvriers ont pris la pose pour un cliché. La cinquième est un photogramme du film montrant les ouvriers sur le chemin de l'usine. N'importe quel spectateur peut remarquer la différence de grain entre les premières images, c'est-à-dire les clichés d'époque, et celle-ci, qui, bien qu'étant un noir et blanc assez délavé, présente une définition nettement supérieure. Par la suite, la pellicule continue d'imbriquer les images tirées du film et les images d'archive, poursuivant sa présentation des différents acteurs de la «lutte des classes » : les ouvriers, l'intellectuel venu de Gênes les politiser, l'armée, les grands bourgeois turinois (qui, à l'époque, ne se déplacent pas encore en automobile mais en carrosse, comme semblent ironiquement vouloir le rappeler les plans 16-17). La dernière image est une photo probablement tirée de la presse écrite, montrant une rue de Turin noircie par une foule de manifestants. Un fondu au noir, très bref, nous introduit au premier plan de la première séquence: c'est une vue sur la cour d'un lotissement ouvrier, au petit matin, traversée de droite à gauche, au pas de course, par une femme vêtue de noir. L'inscription en caractères blancs "Turin à la fin du XIX siècle » apparaît au bas de l'écran après un léger traveling de bas en haut. Il s'agit d'un simple plan de situation, puisque l'action se transfère immédiatement à l'intérieur d'une demeure ouvrière.

11 La particularité de ce générique, ce sont les mouvements de caméra sur certaines images, généralement des zooms avant ou arrière, dont la trajectoire est animée tantôt par de légers mouvements diagonaux, tantôt par des travellings verticaux. Ces mouvements donnent aux plans un statut hybride, celui d'images fixes cinématographiées, portant donc une marque forte de filmicité. La musique, pour une fois, n'a rien d'orchestral : c'est une chanson ouvrière à équivoque, accompagnée à la guitare et à l'accordéon, qui renvoie directement au milieu social dans lequel va se dérouler l'essentiel de l'action. Il est vrai que la musique des génériques de La Grande Pagaille et de La Marche sur Rome pourrait tout aussi bien être exécutée par un orchestre, et qu'elle renvoie là aussi, de façon presque aussi directe, aux éléments du scénario : la guerre, la vie militaire ou paramilitaire, en soulignant leur côté fanfaronnant et « déglingué ». 
IV.

Synopsis du générique d'ouverture de I compagni (109")16

\begin{tabular}{|c|c|c|c|}
\hline $\begin{array}{l}\text { Numéro } \\
\text { du plan }\end{array}$ & $\begin{array}{l}\text { Grosseur } \\
\text { de plan }\end{array}$ & $\begin{array}{l}\text { Description de l'image fixe } \\
\text { et éventuels mouvements de caméra }\end{array}$ & $\begin{array}{l}\text { Nature de } \\
\text { l'image originale }\end{array}$ \\
\hline 1 & $\mathrm{PDE}>\mathrm{PE}$ & $\begin{array}{l}\text { de la place San Carlo, avec ses deux églises baroques } \\
\text { jumelles (et la statue équestre d'Emmanuel Philibert); } \\
\text { zoom arrière sur la place, englobant les arcades et un } \\
\text { deuxième trolley }\end{array}$ & $\begin{array}{l}\text { Photo d'époque } \\
\text { (carte postale) }\end{array}$ \\
\hline 2 & $\mathrm{PE}$ & $\begin{array}{l}\text { sur le hangar et les quais de la gare des trains (vue } \\
\text { dans l'axe longitudinal) }\end{array}$ & $\begin{array}{l}\text { Photo d'époque } \\
\text { (carte postale) }\end{array}$ \\
\hline 3 & $\mathrm{PE}>\mathrm{PG}$ & $\begin{array}{l}\text { sur le Pô et ses berges à la hauteur des jardins du } \\
\text { Valentino ; zoom arrière }\end{array}$ & $\begin{array}{l}\text { Photo d'époque } \\
\text { (carte postale) }\end{array}$ \\
\hline 4 & $\mathrm{PE}>\mathrm{PG}$ & $\begin{array}{l}\text { sur la via Po dans (vue dans l'axe longitudinal) : en } \\
\text { arrière-plan, l'église de la Gran Madre; zoom arrière } \\
\text { jusqu'à l'embouchure de la rue, côté Piazza Castello }\end{array}$ & $\begin{array}{l}\text { Photo d'époque } \\
\text { (carte postale) }\end{array}$ \\
\hline 5 & $\mathrm{PDE}>\mathrm{PM}$ & $\begin{array}{l}\text { sur un atelier d'usine : une vingtaine d'ouvriers sont } \\
\text { tournés vers l'objectif; zoom avant sur une dizaine } \\
\text { d'ouvriers }\end{array}$ & Photo d'archive \\
\hline 6 & PDD & $\begin{array}{l}\text { sur une cheminée d'usine; zoom arrière sur les } \\
\text { ouvriers (vus de dos) se rendant à l'usine: on y } \\
\text { distingue la présence de nombreuses femmes }\end{array}$ & $\begin{array}{l}\text { Photogramme } \\
\text { du film ou } \\
\text { photographie de } \\
\text { plateau }\end{array}$ \\
\hline 7 & $\mathrm{PM}>\mathrm{PR}$ & $\begin{array}{l}\text { sur une famille en intérieur (il s'agit manifestement } \\
\text { d'un taudis insalubre, avec des murs faits de planches } \\
\text { de bois et de chiffons. Au premier plan, une petite fille } \\
\text { accroupie, le visage tourné vers l'objectif, l'œil vif. Au } \\
\text { second plan, de profil, une femme tenant un enfant } \\
\text { dans l'un de ses bras et s'affairant de l'autre autour du } \\
\text { poêle à cuisson. Le bébé emmitouflé a lui aussi l'œil } \\
\text { tourné vers la caméra. Au dernier plan, deux autres } \\
\text { enfants plus grands, assis. Zoom avant sur la petite } \\
\text { fille, les deux autres enfants demeurant toujours dans } \\
\text { le cadre }\end{array}$ & $\begin{array}{l}\text { Photogramme } \\
\text { du film ou } \\
\text { photographie de } \\
\text { plateau }\end{array}$ \\
\hline 8 & $\mathrm{PR}>\mathrm{PM}$ & $\begin{array}{l}\text { sur foule d'ouvriers ; zoom arrière (en plongée) sur ce } \\
\text { qui apparaît alors comme une réunion syndicale à } \\
\text { l'intérieur d'un local à l'apparence miséreuse }\end{array}$ & $\begin{array}{l}\text { Photogramme } \\
\text { du film ou } \\
\text { photographie de } \\
\text { plateau }\end{array}$ \\
\hline
\end{tabular}




\begin{tabular}{|c|c|c|c|}
\hline 9 & PM & $\begin{array}{l}\text { (intérieur) sur des ateliers d'usine: à gauche du } \\
\text { passage en planche de bois, des femmes assises se } \\
\text { tournant vers l'objectif pour la pose; à droite, des } \\
\text { hommes }\end{array}$ & Photo d'archive \\
\hline 10 & PM & $\begin{array}{l}\text { (extérieur) sur des hommes et des femmes occupés à } \\
\text { remplir sacs et paniers, champ fermé par une palissade } \\
\text { en bois; zoom arrière englobant, au premier plan, } \\
\text { deux figures d'adolescents (un garçon e une fille), vus } \\
\text { de dos }\end{array}$ & $\begin{array}{l}\text { Photogramme } \\
\text { du film ou } \\
\text { photographie de } \\
\text { plateau }\end{array}$ \\
\hline 11 & PM & $\begin{array}{l}\text { sur les ouvriers en train de se nourrir (avec profondeur } \\
\text { de champ sur l'intérieur de l'usine) }\end{array}$ & $\begin{array}{l}\text { Photogramme } \\
\text { du film ou } \\
\text { photographie de } \\
\text { plateau }\end{array}$ \\
\hline 12 & $\mathrm{PDD}>\mathrm{PA}$ & $\begin{array}{l}\text { sur des mains d'homme serrant un pain; zoom arrière } \\
\text { sur la figure du professeur Sinigaglia (Marcello } \\
\text { Mastroianni) }\end{array}$ & $\begin{array}{l}\text { Photogramme } \\
\text { du film ou } \\
\text { photographie de } \\
\text { plateau }\end{array}$ \\
\hline 13 & PM & $\begin{array}{l}\text { sur des ouvriers assis sur des rails pour la pause } \\
\text { déjeuner, centrage sur une figure féminine; zoom } \\
\text { arrière englobant au premier plan un ouvrier } \\
\text { corpulent (Pautasso) buvant du vin à la bouteille. } \\
\text { Derrière lui, assise elle aussi, une jeune femme (sa } \\
\text { fille). Sont également visibles, en arrière-plan } \\
\text { brumeux/flouté, des dizaines d'autres ouvriers }\end{array}$ & $\begin{array}{l}\text { Photogramme } \\
\text { du film ou } \\
\text { photographie de } \\
\text { plateau }\end{array}$ \\
\hline 14 & $\mathrm{PDD}>\mathrm{PR}$ & $\begin{array}{l}\text { sur une tranche de pain ; travelling de bas en haut sur } \\
\text { un enfant ouvrier (Omero) }\end{array}$ & $\begin{array}{l}\text { Photogramme } \\
\text { du film ou } \\
\text { photographie de } \\
\text { plateau }\end{array}$ \\
\hline 15 & $P R>G P$ & $\begin{array}{l}\text { sur couple groupe de bourgeois en extérieur; zoom } \\
\text { avant sur le visage d'un homme barbu en haut-de- } \\
\text { forme }\end{array}$ & Photo d'archive \\
\hline 16 & PDE & sur carrosse et personnages en habits bourgeois & Photo d'archive \\
\hline 17 & $\mathrm{PR}>\mathrm{PDE}$ & $\begin{array}{l}\text { sur un officier faisant le salut martial ; zoom arrière } \\
\text { sur une parade de cavalerie (corso Vittorio Emanuele } \\
\text { II ?) }\end{array}$ & Photo d'archive \\
\hline 18 & PM & $\begin{array}{l}\text { sur une scène chorale d'extérieur; un zoom arrière } \\
\text { laisse reconnaître la scène de la bagarre entre } \\
\text { grévistes et « jaunes » à la gare ferroviaire }\end{array}$ & $\begin{array}{l}\text { Photogramme } \\
\text { du film }\end{array}$ \\
\hline
\end{tabular}




\begin{tabular}{|l|l|l|l|}
\hline 19 & PD>PM & $\begin{array}{l}\text { sur une pancarte («Basta con i mutilati del lavoro»: } \\
\text { "Stop aux mutilés du travail”); zoom arrière sur le } \\
\text { groupe de grévistes et apparition de deux autres } \\
\text { pancartes (la première illisible, sur la seconde, en } \\
\text { caractères plus gros, on peut lire les mots « Poco pane } \\
\text { »: «Peu de pain »). }\end{array}$ & $\begin{array}{l}\text { Photogramme } \\
\text { du film }\end{array}$ \\
\hline
\end{tabular}

[Légende du tableau ci-dessus : $P D D=$ plan de détail $; P R=$ plan rapproché $; P A=$ plan américain ; $\mathrm{PM}=$ plan moyen $; \mathrm{PDE}=$ plan demi-ensemble $; \mathrm{PE}=$ plan d'ensemble $; \mathrm{PG}=$ plan général]

\section{V.}

Selon les dictionnaires, le verbe "composer» implique l'idée d'assemblage, d'agencement, d'arrangement, d'organisation de parties: on retrouve ces concepts associées à la notion de composite. Par extension de sens, le verbe « composer » signifie aussi s'accommoder, traiter, transiger, voire pactiser, et emporte alors avec lui l'idée de "concession", apparemment étrangère et même antinomique par rapport au « composite » de l'art contemporain, où l'hétéroclite, l'hétérogène, le fragmentaire sont l'indice d'une volonté de transgression ou de dépassement esthétique, excluant toute notion de « compromission». Sur ces prémisses, il pourrait sembler vain de rechercher, dans le cinéma narratif/commercial de l'époque considérée, une quelconque trace de " composite », sinon - comme on l'a fait jusqu'ici - sur l'axe syntagmatique, c'est-à-dire dans l'agencement horizontal d'images. Même le générique des Monstres (1962) - un film qui n'appartient pas au corpus envisagé mais qui joue la carte de la composition au niveau scénaristique ${ }^{17}$ - bien qu'il fasse appel, dans sa recherche d'hyper-modernité, à des éléments inspirés du minimalisme de la peinture abstraite et du design domestique, ne se hasarde pas dans le «composite", entendu au sens d'un affichage de l'acte de d'assemblage. D'abord parce que le cinéma courant est encore, au début des années 60 , un cinéma de la transparence et, en second lieu, parce que la télévision, c'est-à-dire la vidéo, n’a pas encore fait école. S'agissant de la création de génériques de plus en plus originaux et "artistiques", nous sommes en plein dans l'enfance de l'art, dans sa phase expérimentale ${ }^{18}$. Seulement quelques années plus tard, en 1966, on connaîtra le générique «collage » de L'Armée Brancaleone de Monicelli, où l'on voit les têtes des personnages, découpées dans les photos de plateau des comédiens, dodeliner sur des corps minuscules de dessin animé. Dans les génériques étudiés, la musique fonctionne certes par agencement d'images sonores ; mais le son de trompette présent sur plusieurs d'entre eux est purement référentiel - fonctionnel, pourrait-on dire - , il renvoie à la vie militaire, de même que celui du cor et que les roulements de tambour. Et, pour ce qui relève de l'iconique, l'assemblage et la combinaison s'opèrent, le plus souvent, bien en amont. Il est toutefois une dimension où l'art de la«comédie historique» peut bien être défini comme pluriel : c'est dans la multiplication des intentions, ainsi que dans le caractère collectif de l'écriture ( $L a$ Marche sur Rome est écrit à douze mains ${ }^{19}$ ); il l'est, également, dans la choralité des sujets de l'action, en particulier chez Monicelli dont on sait le combat qu'il eut à mener pour imposer un casting et un traitement visuel de ses acteurs conforme à la représentation qu'il avait imaginée pour ses personnages de "perdants » de l'Histoire ${ }^{20}$. 
VI.

13 Mario Monicelli est le cinéaste qui a le plus contribué à alimenter le filon des comédies historiques en Italie, et ce, bien au delà de la période envisagée ${ }^{21}$. Il faut lui reconnaître en outre le mérite d'avoir participé activement, à partir du Pigeon (1958), qui amorce la transformation de la comédie et des filons qui la composaient jusqu'alors (film comique d'inspiration théâtrale et de matrice régionale, comédie sentimentale, comédie de mœurs...) en « comédie à l'italienne », à la création d'un super-genre, d'un genre hybride, "ouvert», capable d'en intégrer de nombreux autres (film de guerre, film d'action, fresque historique...), faisant par là confluer dans la réalisation des œuvres tous les savoir-faire et toutes les forces vives du cinéma italien ${ }^{22}$. Ses deux films pris en considération sont en outre particulièrement représentatifs d'un cinéma qui, à nouveau depuis le Néoréalisme et dans son sillage moral, «se sent partie prenante d'un projet politique tendant à modifier la réalité dans un sens progressiste ${ }^{23} »$. On a pu parler d'un « projet Monicelli »-expression qui naguère, appliquée à un spécialiste de la comédie, aurait pu étonner - , soulignant la dimension programmatique qui semble sous-tendre l'œuvre du réalisateur toscan de La Grande Guerre (voire du Pigeon) jusqu'aux Camarades ${ }^{24}$. Les scénaristes ont également joué un rôle de tout premier plan dans la réussite des films ainsi que dans la « réforme goldonienne » que la comédie subit en Italie au passage entre les deux décennies. Agenore Incrocci et Furio Scarpelli, scénaristes attitrés de Monicelli pendant une décennie, ont collaboré à l'écriture des quatre comédies étudiées, devenues des classiques du genre, ainsi qu'au scénario des Meilleurs Ennemis (cf. infra, Filmographie). Selon Maurizio Grande, l'observation de leurs scripts fait ressortir de quelle manière le problème de la composition est ressenti comme la question essentielle de l'écriture pour l'écran. Les scénarios d'Age et Scarpelli sont de véritables textes amphibiens où la composition donne lieu à une écriture multiplanaire qui organise les principes dramaturgiques et narratifs en une orchestration audiovisuelle de la page ${ }^{25}$. (tdr)

C'est spécifiquement au plan de la composition que l'on relève tous les traits distinctifs de leur écriture cinématographique : la modulation du dramatique et du comique, la tonalité épique de la comédie, qui souvent (et plus particulièrement chez Monicelli) se réfère au genre picaresque, à son tour tributaire d'Apulée. De ce point de vue, le macchiettismo, reproche que la critique transalpine a régulièrement adressé à la comédie nationale- et qu'on l'on pourrait traduire en français, en empruntant les mots de Frédy Buache ${ }^{26}$ et en conservant la connotation péjorative de l'italien, par «composition par tableautins » assume une toute autre dimension, qu'il ne faudrait hésiter, avec le recul, à définir comme constitutive du genre lui-même, un véritable «modèle paradigmatique ${ }^{27}$ » aussi bien au plan des contenus qu'au plan de la forme. C'est bien d'ailleurs ce type de construction qui d'abord permet d'alterner la focalisation sur l'individu à la focalisation sur le groupe social, conférant au récit un caractère dominant de choralité, en dépit de la présence parfois encombrante de vedettes du calibre d'Alberto Sordi, de Vittorio Gassman, d'Ugo Tognazziou de Marcello Mastroianni pour ne citer que les Italiens. Tout aussi considérable pour la réussite économique et artistique de ces œuvres est en effet l'apport des comédiens, une variante à l'évidence moins programmable et un matériau assurément moins malléable lorsqu'il s'agit d'acteurs de cette personnalité. La naissance (contemporaine et presque consubstantielle à celle du genre rénové), d'un star system prêt à accéder à la notoriété internationale et sa mise à contribution participent aussi de cette 
gageure qui consiste à harmoniser des exigences- spectaculaires, tonales, de format commercial, éthiques - a priori divergentes.

\section{Filmographie : comédies sur toile de fond historique (1959-1963)}

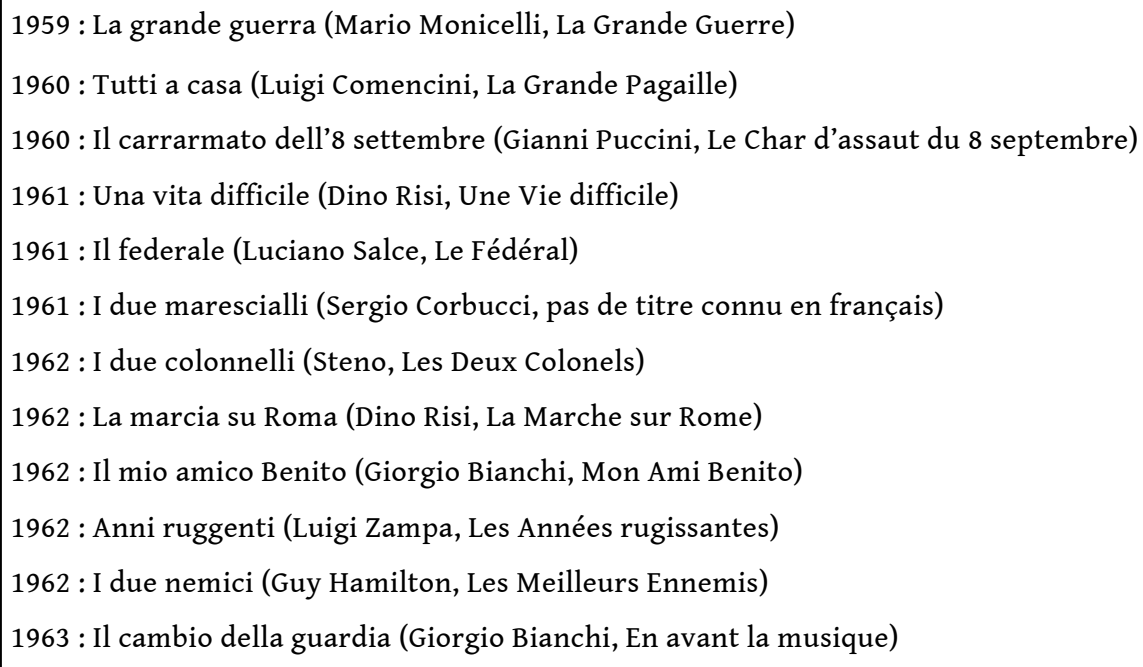

\section{BIBLIOGRAPHIE}

BALDELLI, Pio, Il problema della narrativa cinematografica popolare, in Mario Monicelli, I compagni. Comicità e tragedia nei primi scioperi operai (sld Pio Baldelli), Cappelli, Bologne, 1963. BRUNETTA, Gian Piero, Storia del cinema italiano (1905-2003), Einaudi, Turin, 2003.

BRUNO, Michela, La storia dei titoli di testa, consulté le 29/10/2010 à l'adresse [http:// www.rainboway.it/immagini/passions/filmtitles/titolitesta.html]

Générique \& cinéma. $1^{\text {er }}$ site français dédié aux génériques des films, consulté le 29/10/2010 à l'adresse [http://www.generique-cinema.net/]

GILI, Jean A., La Comédie italienne, Henri Veyrier, Paris, 1985.

GRANDE, Maurizio, La commedia all'italiana (sld Orio Caldiron), Bulzoni, Rome, 2003.

PORTELLI, Aurélien, L'Histoire contemporaine de l'Italie sur grand écran : pouvoir et société dans le cinéma historique italien de 1954 à 2006, Thèse de doctorat soutenue à l'Université Nice Sophia Antipolis, 2007.

RE, Valentina, Ai margini del film. Incipit e titoli di testa, Campanotto, Udine, 2006.

Récits et genres historiques, Aude Deruelle et Jean-Paul Aubert (sld), Cahiers de Narratologie $n$ •15, consulté le 29/10/2010 [http://revel.unice.fr/cnarra/index.html ?id =676]. 
TYLSKI, Alexandre (sld), Les cinéastes et leurs génériques, L’Harmattan, 2008.

\section{NOTES}

1. Alessandro Blasetti, Le cinéma : art composite ou art spécifique, exposé préliminaire à la Conférence Internationales des Artistes organisée par l'UNESCO, à Venise, en 1952. Texte paru en traduction française dans « Art » n 6, Paris, 11 août 1952.

2. Les résultats de ces recherches sont aisément accessibles sur un site web francophone dédié, comportant une bibliographie/sitographie multilingue (http://www.generique-cinema.net).

3. Alexandre Tilsky, Le Générique au cinéma: histoire et fonctions d'un fragment hybride, Presses universitaires du Mirail, Toulouse, 2009.

4. Enrico Giacovelli, Breve storia del cinema comico in Italia, Lindau, Torino, 2002.

5. L'expression est de Jean A. Gili, qui dans sa monographie y consacre un paragraphe à part. Cf. Jean A. Gili, La Comédie italienne, Henri Veyrier, Paris, 1985.

6. Le succès des films de reconstruction de l'époque du muet, le plus souvent à caractère historico-mythologique, est l'exemple précoce d'une prérogative longtemps conservée par le cinéma italien au cours du XXe siècle. C'est en Italie que dans les années 10 le long métrage s'imposa comme le format filmique prédominant,comme une conséquence de la volonté d'intégrer dans les œuvres un nombre toujours plus grand d'éléments scénaristiques, scénographiques, actoriels.

7. Mario Monicelli, Autoritratto, Edizioni Polistampa, Firenze, 2002.

8. Ibid.

9. "Gli italiani sono disposti ad affrontare qualsiasi argomento a patto di poterne ridere". L'expression est de Masolino D'Amico, in La commedia all'italiana, Mondadori, Milan, 1985. On notera au passage, pour souligner ce caractère de typicité, que le cinéma de Federico Fellini l'auteur par antonomase, le cinéaste à scandale - n'est que très rarement exempt de comicité.

10. Gian Piero Brunetta, Storia del cinema italiano, p. 210.

11. En 1960, 160 films sont produits en Italie, dont 66 coproductions (et, parmi celles-ci, 61 avec la France).

12. Les sources littéraires du film sont multiples : de la nouvelle Les Deux Amis de Maupassant au roman autobiographique d'Emilio Lussu Un An sur les hauteurs (Un anno sull'altipiano, 1938) qui quelques années plus tard sera porté sur l'écran par Francesco Rosi sous le titre Les Hommes contre (Uomini contro, 1970). De nombreux épisodes et personnages du film de Monicelli semblent eux aussi directement inspirés du livre qui est considéré comme l'un des témoignages les plus authentiques et poignants de la première guerre mondiale; les auteurs de La Grande Guerre ne cachèrent jamais qu'il était l'une de leurs principales sources documentaires: cependant, Emilio Lussu (député antifasciste, membre à l'époque du Parti Socialiste Italien), refusa de voir son nom paraître au générique du film.

13. Ce qui pose au passage la question de la catégorisation de ces films: dans le Dictionnaire mondial des films de B. Rapp et J.-C. Lamy, par exemple, La Grande Pagaille est classé « drame », de même que La Grande Guerre et Les Camarades. Les Années difficiles est défini « drame social », alors que Les Années rugissantes (L. Zampa, 1962) est considéré comme une « comédie ».

14. Difficile de penser que la comédie, sur les prémisses de La Grande Guerre, ne s'empare pas des potentialités tragicomiques d'un tel sujet. Sur les 12 comédies citées en filmographie, On peut noter au passage qu'en 1963 le scénariste Age considère La Grande Pagaille comme sa plus belle réussite. Cf. Baldelli (1965, p. 56)

15. Le Milanais Giovanni Busacca (V. Gassman) et le Romain Oreste Jacovacci (Alberto Sordi). Le filon de la « comédie historique » se nourrit d'une forte intertextualité, d'un « effet corpus » qui lie les films les uns aux autres, au-delà des histoires racontées, des références historiques et des 
sources historiographiques parfois communes. Dans La Marche sur Rome, où Gassman joue le personnage d'un Romain, le rôle du settentrionale, revient «naturellement » au Mantouan Ugo Tognazzi.

16. Ces images peuvent être visionnées sur l'Internet à l'adresse http://www.youtube.com/ watch?v=QWlZVjzF79I\&feature=related

17. Ce film sketches comporte 17 épisodes dans sa version intégrale. Mosaïque sociologique de l'Italie du «boom», il affiche 6 prestigieuses signatures au tableau des scénaristes: Agenore Incrocci, Ruggero Maccari, Elio Petri, Dino Risi (réalisateur du film), Furio Scarpelli et Ettore Scola.

18. Selon la périodisation historique des génériques d'ouverture proposée par Michela Bruno, cf. Bibiographie.

19. Hormis la participation de Dino Risi qui en est le réalisateur. On y voit apparaître, sur le même plan, les noms d'Age (Agenore Incrocci), de Furio Scarpelli, d'Ettore Scola, de Ruggero Maccari, de Sandro Continenza et de Ghigo de Chiara. Un record est sans doute atteint avec Il carrarmato dell' 8 settembre: on peut compter au registre des scénaristes neuf signatures, certaines fort prestigieuses aujourd'hui et à différents titres, comme celles de Pier Paolo Pasolini, Rodolfo Sonego, Elio Petri et Goffredo Parise.

20. On sait notamment qu'afin de réaliser le projet des Camarades selon les propres exigences artistiques des auteurs, Monicelli fit appel à une maison de production yougoslave, renonçant aux capitaux qui lui étaient offerts localement.

21. A commencer par les deux films du diptyque de l'Armata Brancaleone (1966 et 1970), puis Il marchese del Grillo (1981), I Picari/Les Picares (1988), Rossini Rossini (1991) et enfin Le rose del deserto (2010), adapté d'un roman de Mario Tobino (Il deserto della Libia, 1952) dont Dino Risi avait déjà, en 1985, fait une adaptation cinématographique interprétée par Coluche et Beppe Grillo et intitulée Le Fou de guerre/Scemo di guerra.

22. Ainsi, selon Gian Piero Brunetta, « cette force qui unit, comme cela n'était sans doute jamais arrivé auparavant, tous les niveaux du système, [...] cette circulation des techniciens, des scénaristes, des décorateurs, des costumiers, des compositeurs, des acteurs, des comparses, des cameramen » $(\mathrm{tdr})$ mériterait une plus grande attention de la part des chercheurs » $(\mathrm{tdr})$, in Brunetta (2003), p.212.

23. Brunetta (2003), p. 218.

24. Stefano Della Casa, Mario Monicelli, Il Castoro 124, La Nuova Italia, Firenze, juillet-août 1986, p. 9-13.

25. "Age et Scarpelli : l'épopée du clair-obscur », in M. Grande (2003), p. 235.

26. Frédy Buache, Le cinéma italien (1945-1990), Éditions l'Âge d'Homme, Lausanne, 1992, dans sa recension à La Grande Guerre. La critique du film est globalement plutôt négative, apparemment sur la ligne de l'école critique italienne, d'empreinte marxiste. Elle attire cependant l'attention du spectateur non-italophone sur « le choc des particularismes régionaux, la réunion d'hommes de conditions et de provinces diverses qui se trouvent engagés dans une commune action non exempte de truculence malgré son caractère douloureux et angoissant. Cette situation particulière n'apparaît qu'à travers le dialogue qui, dans la langue originale, fait appel aux multiples ressources vivantes des dialectes italiens. » Un autre aspect de cet « art de la composition » sociologique qui confère à Monicelli et à ses scénaristes un titre de mérite supplémentaire dans l'invention de la « comédie à l'italienne ".

27. Cf. M. Grande (2003), p. 236. 


\section{RÉSUMÉS}

Si le générique est, à l'intérieur du film, un lieu privilégié de composition iconique et sonore, le cas de comédies historiques italiennes est particulièrement révélateur de la multiplicité d'intentions qui préside à leur réalisation.

Se è vero che i titoli di apertura di un film costituiscono uno spazio privilegiato di composizione iconica e sonora, quelli delle commedie a sfondo storico sono particolarmente esemplari della molteplicità di intenti che presiede alla loro produzione.

\section{INDEX}

Mots-clés : Cinéma italien, composition, générique, genre comique, relecture historique

\section{AUTEUR}

\section{STEFANO LEONCINI}

CIRCPLES - EA 3159, Université Nice-Sophia Antipolis 